\title{
Gamow-Teller Transitions Starting from Stable and Unstable $p f$-shell Nuclei
}

\author{
Yoshitaka Fujita* \\ Department cf Physics, Osaka University, Toyonaka, Osaka 560-0043, Japan, \\ for the RCNP, Osaka, High Resolution $\left({ }^{3} \mathrm{He}, t\right)$ Collaboration, and \\ for the Valencia, Surrey, Osaka, GSI, Istanbul, Kraków, Legnaro, Leuven, Lund, Madrid, New \\ Delhi, Santiago de Compostela, $\beta$-decay Collaboration \\ E-mail: fujita@rcnp.osaka-u.ac.jp
}

Gamow-Teller (GT) transition is one of the most popular nuclear weak processes caused by the very simple spin-isospin $(\sigma \tau)$ operator. It is of interest not only in the study of nuclear physics, but also in astrophysics; it plays important roles, for example, in supernova-explosion or nuclear synthesis. Relatively limited information can directly be obtained through the study of weak processes, such as $\beta$-decay or neutrino induced reactions. However, it was found that chargeexchange (CE) reactions, such as $(p, n),(n, p),\left({ }^{3} \mathrm{He}, t\right)$ or $\left(t,{ }^{3} \mathrm{He}\right)$, at intermediate incoming energies $(E>100 \mathrm{MeV})$ and at $0^{\circ}$ can selectively excite GT transitions. This is due to the fact that the $\sigma \tau$-type operator plays relatively important roles under these conditions. Therefore, the study of nuclear weak response was extended to the higher-excited region of so-called Gamow-Teller giant resonance (GTGR) with $E_{x}=10 \mathrm{MeV}$ or more. With one-order-of-magnitude improvement of the energy resolution in $\left({ }^{3} \mathrm{He}, t\right)$ measurements at $140 \mathrm{MeV} / n u c l e o n$, fine structures of GT excitations, even those of GTGRs, can now be studied. Determination of GT transition strengths for $p f$-shell nuclei with astrophysical interest is discussed. We show that quantum number "isospin" plays important roles in such studies. A method to derive $B(\mathrm{GT})$ values, in that we combines the strength distribution form the $\left({ }^{3} \mathrm{He}, t\right)$ measurement with the half-life and $Q$-value information from the mirror $\beta$-decay study, is presented. In this analysis, we assume good isospin symmetry of GT transitions in $\pm\left|T_{z}\right| \rightarrow \pm\left|T_{z}-1\right|$ nuclei. This "merged analysis" is powerful in deriving the absolute $B(\mathrm{GT})$ values for $p f$-shell nuclei.

10th Symposium on Nuclei in the Cosmos

July 27 - August 12008

Mackinac Island, Michigan, USA

${ }^{*}$ The $\left({ }^{3} \mathrm{He}, t\right)$ high-energy resolution experiments were performed at RCNP, Osaka, by a large number of collaborators from various institutes. Especially effort from Georg P.A. Berg (Notre Dame), Hirohiko Fujita (Osaka), Yoshihiro Shimbara (Niigata) and Tatsuya Adachi (Osaka) are acknowledged. Leading roles performed by Berta Rubio (Valencia) and William Gelletley (Surrey) in the bata-decay studies are appreciated. The $\left(t,{ }^{3} \mathrm{He}\right)$ reactions were performed at MSU by the group lead by Remco Zegers. 


\section{Introduction}

Weak-interaction processes of $p f$-shell nuclei play important roles in the core-collapse stage of type II supernovae. Therefore, studies of electron capture and $\beta$-decay caused by charged currents and neutrino-nucleus scattering involving neutral currents are of great astrophysical interest $[1,2,3]$. The charged-current processes are dominated by Fermi and Gamow-Teller (GT) transitions. The Fermi strength is concentrated in the transition to the isobaric analog state of the ground state (g.s.) of the mother nucleus (IAS), but our knowledge of the GT transitions is very poor. Therefore, interest in the accurate determination of absolute values and the distribution of GT transition strengths in $p f$-shell nuclei are increasing.

In laboratory experiments, direct information on the GT transition strength $B(\mathrm{GT})$ can be derived from $\beta$-decay measurements. In a $\beta$-decay, the partial half-life $t_{i}$ of the $i$ th GT transition and $t_{\mathrm{F}}$ of the Fermi transition multiplied by the phase-space factor ( $f$-factor) are related, respectively, to the $B(\mathrm{GT})$ and the reduced Fermi transition strength $B(\mathrm{~F})$ as

$$
f t_{i}=K / \lambda^{2} B(\mathrm{GT}) \text { and } f t_{\mathrm{F}}=K / B(\mathrm{~F})\left(1-\delta_{c}\right),
$$

where $K=6147.8(16), \lambda=g_{A} / g_{V}=-1.270(3), \delta_{c}$ is the Coulomb correction factor [4], and $f_{\mathrm{F}}$ and $f_{i}$ are the phase-space factors ( $f$-factors) of the $\beta$-decay to the IAS and to the $i$ th GT state, respectively. The Fermi transition strength has the value $B(\mathrm{~F})=|N-Z|$. On the other hand, the $B(\mathrm{GT})$ values are largely dependent on the structure of each nucleus. Pioneering studies were performed on several far-from-stability $p$ f-shell nuclei (e.g. ${ }^{46} \mathrm{Cr}[5],{ }^{50} \mathrm{Fe}[6],{ }^{54} \mathrm{Ni}[7]$ and $\left.{ }^{58} \mathrm{Zn}[8]\right)$. In these studies, however, $B(\mathrm{GT})$ values were derived for at most a few low-lying states with large uncertainties. Note that the study of the feeding to higher excited states in $\beta$-decay is difficult, because the $f$-factor decreases rapidly with the excitation energy.

Charge-exchange (CE) reactions can access analogous GT transitions without the $Q$-value limitation. In particular, CE measurements performed at angles around $0^{\circ}$ and intermediate beam energies ( $E>100 \mathrm{MeV} /$ nucleon) were shown to be good probes of GT transition strengths owing to the close proportionality between the cross sections at $0^{\circ}$ and the $B(\mathrm{GT})$ values [9]

$$
\frac{d \sigma_{\mathrm{CE}}}{d \Omega}\left(0^{\circ}\right) \simeq \hat{\sigma}^{\mathrm{GT}}\left(0^{\circ}\right) B(\mathrm{GT}),
$$

where $\hat{\sigma}^{\mathrm{GT}}\left(0^{\circ}\right)$ is a unit cross section for the GT transition at $0^{\circ}$. Therefore, if a "standard $B(\mathrm{GT})$ value" is obtained for a mass $A$ system from a $\beta$-decay study, the $B(\mathrm{GT})$ values can be determined even for the transitions to higher excitation energies.

At the high energy-resolution facility of RCNP, Osaka, consisting of RCNP Ring cyclotron [10], a high-dispersive beam line "WS course" [11] and a high-resolution magnetic spectrometer "Grand Raiden" [12], precise beam matching techniques were applied [13, 14, 15] for the $\beta^{-}$-type $\left({ }^{3} \mathrm{He}, t\right)$ $\mathrm{CE}$ reaction at an intermediate beam energy of $140 \mathrm{MeV} /$ nucleon. As a result, in comparison to the pioneering $(p, n)$ work, nearly one order-of-magnitude better resolution $(\Delta E \leq 30 \mathrm{keV})$ has been achieved. Figure 1 shows spectra at different resolutions. A significant improvement in the energy resolution that has been achieved in the past twenty years can be well seen. With a high energyresolution, fine structures have been observed even in the so-called GT giant resonance (GTGR) region of $E_{x}=7-12 \mathrm{MeV}$. We recognise that the GTGR in ${ }^{58} \mathrm{Cu}$ that was observed as a bump-like structure [16] consists of many individual states on a smooth continuum [17]. 


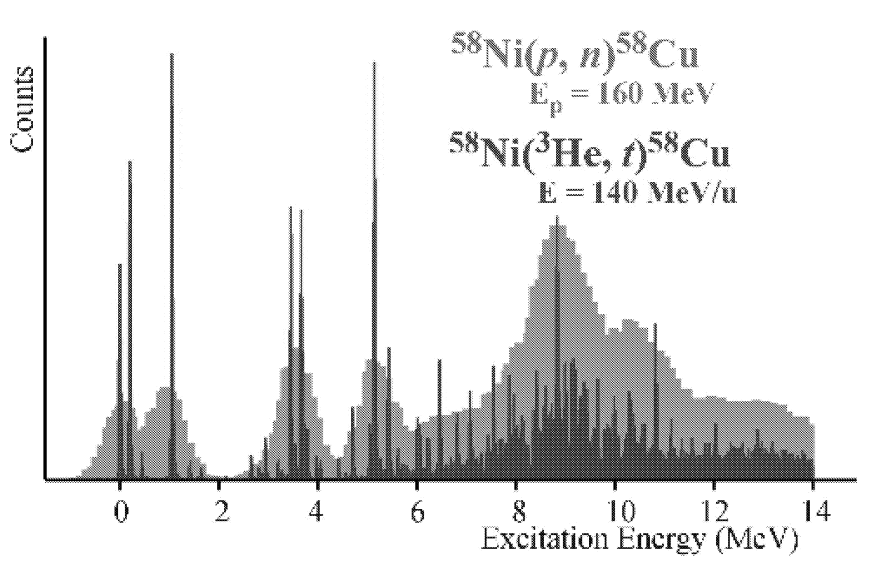

Figure 1: Energy spectra of chargeexchange reactions at $0^{\circ}$. The broad spectrum is from ${ }^{58} \mathrm{Ni}(p, n){ }^{58} \mathrm{Cu}$ reaction measured in 1980's [16]. In the recent ${ }^{58} \mathrm{Ni}\left({ }^{3} \mathrm{He}, t\right){ }^{58} \mathrm{Cu}$ reaction [17] fine structure and sharp states have been observed up to the excitation energy of $13 \mathrm{MeV}$. The proton separation energy $\left(S_{p}\right)$ is at 2.87 MeV. A increase of continuum is observed above $E_{x}=6 \mathrm{MeV}$.

High energy-resolution of spectra makes it possible to study transition strengths for individual GT states. Under the concept of "isospin symmetry" described below, these strengths can be compared directly with those of analogous transitions that can be studied by inelastic scatterings, $\gamma$ decays as well as by mirror $\beta^{+}$decays.

\section{Isospin in nuclei and the validity of the proportionality}

Under the assumption that isospin $T$ is a good quantum number, an analogous structure is expected for nuclei with the same mass $A$ but with different $T_{z}$, i.e., isobars (see e.g. Ref. [18]), where $T_{z}$ is the $z$ component of isospin $T$ defined by $T_{z}=(N-Z) / 2$. The corresponding states in isobars are called analog states and are expected to have the same nuclear structure. Various transitions connecting corresponding analog states are called analogous transitions and have corresponding strengths. As an example, the isospin symmetry structure and analogous transitions are shown for the isobaric systems with $A=26,50$ and 58 in Fig. 2. The thick arrows show pairs of analogous transitions that are studied by the $\left({ }^{3} \mathrm{He}, t\right)$ reaction and the $\beta^{+}$decay. Therefore, the close proportionality given by Eq. (1.2) can be examined by comparing the GT transition strengths observed in the $\left({ }^{3} \mathrm{He}, t\right)$ reaction with those from mirror $\beta^{+}$decay in multiple pairs of analogous transitions. Strengths were compared for analogous GT transitions starting from g.s. of $T_{z}= \pm 1$, $A=26$ nuclei $\left({ }^{26} \mathrm{Mg}\right.$ and $\left.{ }^{26} \mathrm{Si}\right)$ to the $T_{z}=0$ nucleus ${ }^{26} \mathrm{Al}[19,20]$. Such study was also performed for $T_{z}= \pm 1 / 2$ mirror nuclei with $A=27\left({ }^{27} \mathrm{Al}\right.$ and $\left.{ }^{27} \mathrm{Si}\right)[21]$ and $A=23\left({ }^{23} \mathrm{Na}\right.$ and $\left.{ }^{23} \mathrm{Mg}\right)$ [22]. As a result, a proportionality of $\approx 3-10 \%$ has been seen for transitions with an $L=0$ nature and for values of $B(\mathrm{GT}) \geq 0.04$. Therefore, by using the unit GT cross section $\hat{\sigma}^{\mathrm{GT}}\left(0^{\circ}\right)$ for specific $A$ system determined by using the $\beta$-decay $B(\mathrm{GT})$ values, reliable GT strengths can be obtained for the transitions up to highly excited states.

A method using the absolute $\left({ }^{3} \mathrm{He}, t\right)$ cross sections as a means to determine absolute $B(\mathrm{GT})$ values is also proposed [23]. It was suggested that the mass dependence of the unit GT cross section at momentum transfer $q=0$ can be expressed as $\hat{\sigma}^{\mathrm{GT}}(q=0)=109 / A^{0.65}$.

It should be noted that the properties of CE reactions, mediated by the strong nuclear force, are certainly not identical to $\beta$-decay in which the relevant interaction of the allowed GT transition is purely of $\sigma \tau$ type. Thus the proportionality cannot always be valid when the transition strengths are rather weak. Such an exception was found for a relatively weak GT transition from the g.s. of 
$T_{z}=+1,{ }^{34} \mathrm{~S}$ nucleus to a low-lying state of the $T_{z}=0$ nucleus ${ }^{34} \mathrm{Cl}$. The observed GT transition strength was larger by about $40 \%$ compared to that of the analog transition studied in ${ }^{34} \mathrm{Ar} \rightarrow{ }^{34}$ $\mathrm{Cl} \beta$-decay. A DWBA calculation combined with a shell-model calculation suggested that two major configurations of $\sigma \tau$ interaction contribute destructively to this GT transition and, therefore, minor configurations of Tensor $\tau$ interaction make a larger contribution [24]. In addition, a large ambiguity has been reported for the so-called $j_{<}(=\ell-1 / 2) \rightarrow j<$ type transitions, like $d_{3 / 2} \rightarrow d_{3 / 2}$, in the studies of $(p, n)$ reaction [25].

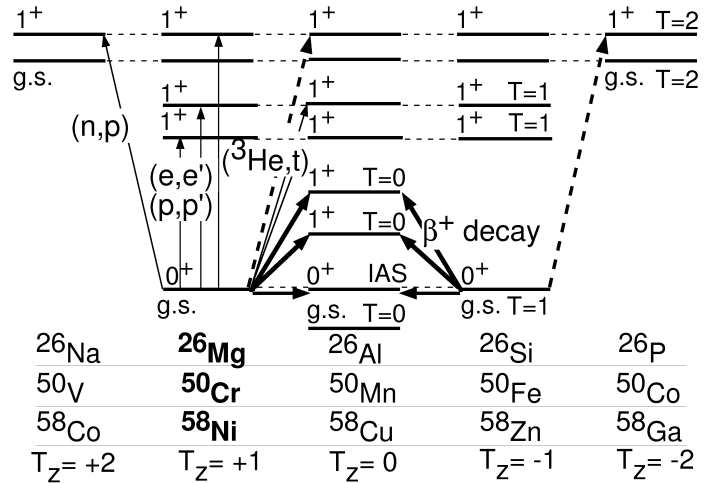

Figure 2: Schematic view of the isospin analog states (connected by horizontal broken lines) and isospin analogous transitions for $T_{z}= \pm 2, \pm 1$ and 0 isobaric nuclei in $A=26,50$ and 58 systems. Stable nuclei ${ }^{26} \mathrm{Mg},{ }^{50} \mathrm{Cr}$ and ${ }^{58} \mathrm{Ni}$ can be the targets in $\mathrm{CE}$ reactions. The Coulomb displacement energies are removed so that the isospin symmetry of the system and that of transitions are put in evidence. The $\left({ }^{3} \mathrm{He}, t\right)$ reaction studies the $T_{z}=+1 \rightarrow 0$ GT transitions and excites $T=0,1$ and 2 states, while the $\beta$-decay studies the analogous GT transitions with $T_{z}=-1 \rightarrow 0$ (thick solid arrows).

\section{Gamow-Teller transitions in $p f$-shell nuclei with astrophysical interest}

Gamow-Teller transitions starting from stable as well as unstable $p f$-shell nuclei are of interest in astrophysics. In the study of $\beta$-decays starting from far-from-stability $T_{z}=-1 p f$-shell nuclei, the determination of feeding ratios to higher excited states is usually difficult, but half-lives can be measured rather accurately. On the other hand, in high resolution $\left({ }^{3} \mathrm{He}, t\right) \mathrm{CE}$ reactions starting from $T_{z}=+1$ nuclei, individual GT transitions up to high excitations can be studied. Assuming the isospin symmetry for the strengths of $T_{z}= \pm 1 \rightarrow 0$ analogous GT transitions, we present a unique "merged analysis" for the determination of absolute $B(\mathrm{GT})$ values.

\subsection{High energy-resolution study of $T_{z}=+1 p f$-shell nuclei}

In the $p f$-shell region, $T_{z}=+1 \rightarrow 0$ transitions can be studied via $\left({ }^{3} \mathrm{He}, t\right)$ reactions on stable $T_{z}=+1$ target nuclei. The obtained spectra for target nuclei ${ }^{42} \mathrm{Ca}[26],{ }^{46} \mathrm{Cr}$ [27], ${ }^{50} \mathrm{Cr}[28]$, ${ }^{54} \mathrm{Fe}[26]$ and ${ }^{58} \mathrm{Ni}[17]$ are shown in Fig. 3.

In the " $T=1$ triplet" system, $T_{z}=+1 \rightarrow 0$ and $T_{z}=-1 \rightarrow 0$ transitions can be studied via a $\left({ }^{3} \mathrm{He}, t\right)$ reaction and a $\beta$-decay from the unstable mirror nucleus, respectively. Assuming that these analogous GT transitions have the same $B(\mathrm{GT})$ values, the $B(\mathrm{GT})$ values from $\beta$-decays $[5,6,7,8]$ can in principle be used as "standard $B(\mathrm{GT})$ values" to determine the $\hat{\sigma}^{\mathrm{GT}}\left(0^{\circ}\right)$ value for each $A$ system. Once the $\hat{\sigma}^{\mathrm{GT}}\left(0^{\circ}\right)$ value is known, the $B(\mathrm{GT})$ values can be determined for the transitions up to highly excited states using Eq. (1.2). However, due to large uncertainties in the $\beta$-decay $B(\mathrm{GT})$ values for the $p f$-shell nuclei, this idea is not practical. 


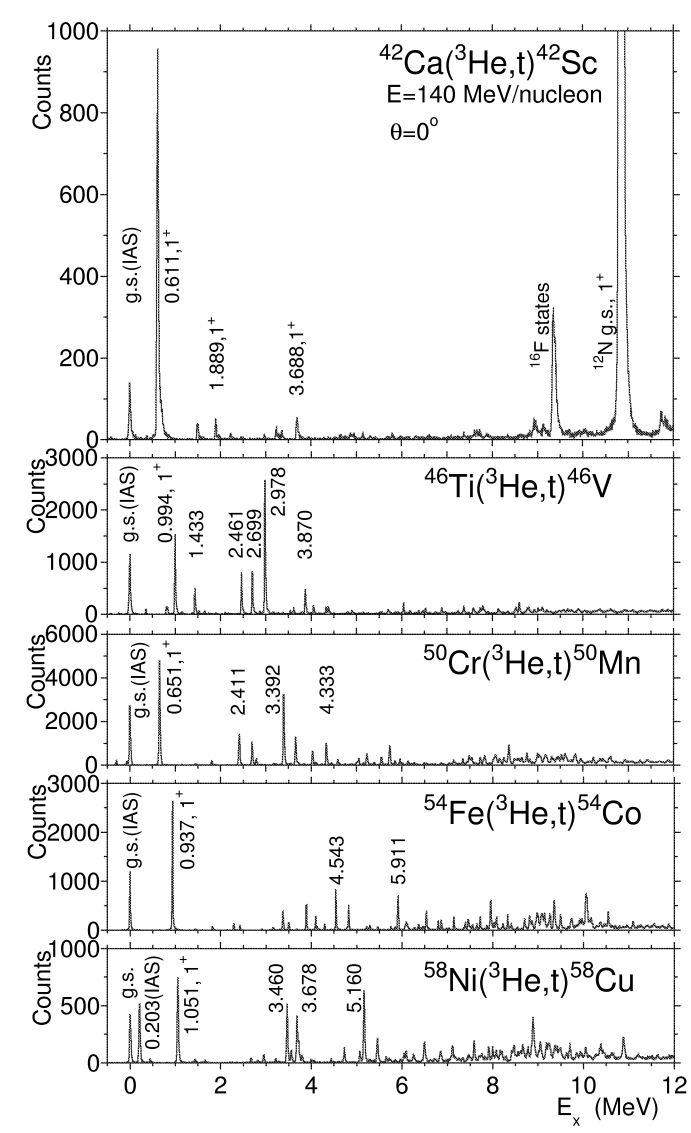

Figure 3: High resolution $\left({ }^{3} \mathrm{He}, t\right)$ spectra for $T_{z}=+1$ target nuclei in $p f$-shell. An energy resolution of $\approx 30 \mathrm{keV}$ is obtained. The vertical scale is normalised by the heights of IAS peaks all having $B(\mathrm{~F})=2$. Thus, peak heights are almost proportional to $B(\mathrm{GT})$. As the mass number $A$ increases, the GT states are more fragmented and more GT strength is found at a higher energy region of 7-12 $\mathrm{MeV}$. The contour of these fragmented states show a bump-like structure. In addition, such bump-like structures identified as GTGRs in pioneering $(p, n)$ measurements [16] are reproduced by smearing these high-resolution spectra with the $(p, n)$ energy resolution of $300-400 \mathrm{keV}$ (see Fig. 1).

\subsection{Merged analysis combining charge-exchange and $\beta$-decay information}

In order to derive more accurate $B(\mathrm{GT})$ values, we introduce the "merged analysis," in which the precise, but relative GT strength distribution from the $\left({ }^{3} \mathrm{He}, t\right)$ reaction is combined with the decay $Q$-value and lifetime from the mirror $\beta$-decay.

In a $\beta$-decay study, the accurate determination of the feeding ratios to higher excited states is more difficult due to smaller $f$-factors [see Eq. (1.1)]. On the other hand, in the $\left({ }^{3} \mathrm{He}, t\right)$ reactions studying the analogous GT transitions, transition strengths that are proportional to $B(\mathrm{GT})$ values (i.e., relative $B(\mathrm{GT})$ values) to these higher excited states can be obtained accurately from the measured cross sections at $0^{\circ}$ [see Eq. (1.2)]. Then the unknown $\beta$-decay feeding ratios can be deduced by combining the $\left({ }^{3} \mathrm{He}, t\right)$ cross sections at $0^{\circ}$ with the $f$-factors that are calculated from the decay $Q$-value. Our idea is to deduce absolute $B(\mathrm{GT})$ values by further combining the knowledge of total half-life $T_{1 / 2}$ of the $\beta$-decay [28].

It should be noted that the inverse of the partial half-life $t_{\mathrm{F}}$ of the Fermi transition and $t_{i} \mathrm{~s}$ of GT transitions are the feeding to the IAS and GT states, respectively. Therefore, the inverse of the half-life $T_{1 / 2}$, i.e., the total transition strength, is the sum of $1 / t_{\mathrm{F}}$ and $1 / t_{i}$ of GT transitions

$$
\left(1 / T_{1 / 2}\right)=\left(1 / t_{\mathrm{F}}\right)+\sum_{i=\mathrm{GT}}\left(1 / t_{i}\right)
$$

Applying Eq. (1.1), one can eliminate both $t_{\mathrm{F}}$ and $t_{i}$, and we get

$$
\frac{1}{T_{1 / 2}}=\frac{1}{K}\left[B(\mathrm{~F})\left(1-\delta_{c}\right) f_{\mathrm{F}}+\sum_{i=\mathrm{GT}} \lambda^{2} B_{i}(\mathrm{GT}) f_{i}\right],
$$


where $f_{\mathrm{F}}$ and $f_{i}$ can be calculated, $B(\mathrm{~F})$ is $|N-Z|$, and the relative strengths proportional to $B_{i}(\mathrm{GT})$ can be studied in the ( ${ }^{3} \mathrm{He}, t$ ) reaction. Therefore, if the total half-life $T_{1 / 2}$ of the $\beta$-decay is known accurately, the relative strengths of $B_{i}(\mathrm{GT})$ studied in the $\left({ }^{3} \mathrm{He}, t\right)$ reaction can be converted into absolute values.

The first analysis was made for the $A=50$ isobaric system (see Fig. 2), because the decay $Q$-value and the total half-life $T_{1 / 2}$ for the ${ }^{50} \mathrm{Fe} \rightarrow{ }^{50} \mathrm{Mn} \beta$-decay $\left[T_{1 / 2}=0.155(11) \mathrm{s}\right.$ and $Q_{\mathrm{FC}}=$ $8.15(6) \mathrm{MeV}][6]$ are the best known among the $T=1$ triplets in the $p f$-shell region. The partial half-lives and thus the feedings, however, were unclear; only the feeding to the first $1^{+}$state at $E_{x}=0.651 \mathrm{MeV}$ had been detected. A $B(\mathrm{GT})$ value of $0.60(16)$ was deduced under the extreme assumption that no feeding took place to higher excited states [6]. On the other hand, in the merged analysis the ratio of unknown feedings up to the higher excitation region of the $T_{z}=-1 \rightarrow 0^{50} \mathrm{Fe}$ $\beta$-decay was deduced by multiplying the ${ }^{50} \mathrm{Cr}\left({ }^{3} \mathrm{He}, t\right)^{50} \mathrm{Mn}$ spectrum [Fig. 4(a)] and the calculated $f$-factor [Fig. 4(b)]. The deduced " $\beta$-decay energy spectrum" is shown in Fig. 4(c).

Putting the value of $T_{1 / 2}$ and the calculated ratio of feedings in Eq. (3.2), absolute GT transition strengths $B(\mathrm{GT})$ were derived for all transitions up to $E_{x}=6 \mathrm{MeV}$ shown in Fig. 4(a) [28]. Owing to the newly deduced feedings to higher excited GT states, the merged analysis showed that the $B(\mathrm{GT})$ value of the first GT state should decrease by about $20 \%$ down to $0.50(13)$. Here the uncertainty originates mainly from the half-life and also the $Q$ value.
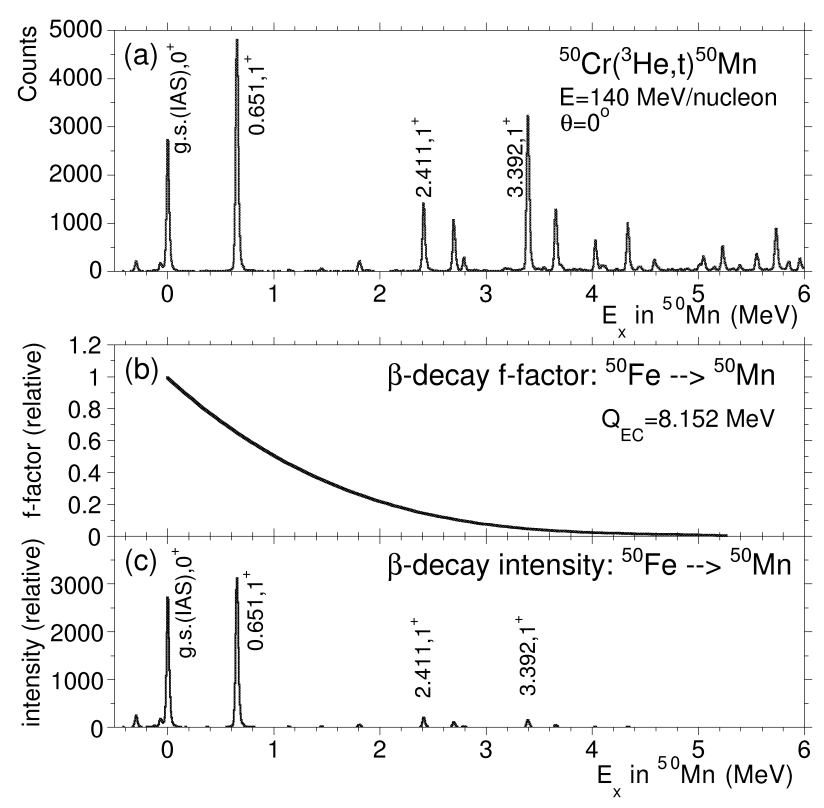

Figure 4: (a) $\mathrm{The}{ }^{50} \mathrm{Cr}\left({ }^{3} \mathrm{He}, t\right)^{50} \mathrm{Mn}$ spectrum for events with scattering angles $\Theta \leq 0.5^{\circ}$. Major $L=0$ states are indicated by their excitation energies in MeV. (b) The $f$-factor for the ${ }^{50} \mathrm{Fe} \beta$-decay, normalised to unity at $E_{x}=0 \mathrm{MeV}$. (c) The estimated ${ }^{50} \mathrm{Fe} \beta$ decay energy spectrum that is obtained by multiplying the ${ }^{50} \mathrm{Cr}\left({ }^{3} \mathrm{He}, t\right)$ spectrum by the $f$-factor. Note that the IAS is stronger in the real $\beta$-decay experiment due to the different ratio of coupling constants for the $\tau$ and $\sigma \tau$-type interactions from the $\left({ }^{3} \mathrm{He}, t\right)$ reaction.

\subsection{Gamow-Teller transitions in the $\beta^{+}$direction}

In $(n, p)$-type reactions, such as $\left(t,{ }^{3} \mathrm{He}\right)$ reaction, on the $T_{z}=+1$ nuclei, $T_{z}=+1 \rightarrow+2 \mathrm{GT}$ transitions in the $\beta^{+}$direction can be studied (the left most thin solid arrow of Fig. 2). These transitions can play important roles in the electron-capture reactions that has a significant influence on the nucleosynthesis as well as neutronization of nuclear mater inside the star. These $T_{z}=+1 \rightarrow$ +2 transitions are analogous with the transitions from g.s. of the same target nucleus to the $T=2$ states that can be studied in the $\left({ }^{3} \mathrm{He}, t\right)$ reaction (thick broken arrow). 
The $\left(t,{ }^{3} \mathrm{He}\right)$ reaction at an intermediate beam energy has been performed at the NSCL, MSU, using a $115 \mathrm{MeV} /$ nucleon secondary triton beam. The outgoing ${ }^{3} \mathrm{He}$ particles were momentum analysed by the S800 spectrometer and an energy resolution of $\approx 200-250 \mathrm{keV}$ has been achieved [29]. The GT strength distribution obtained in the ${ }^{58} \mathrm{Ni}\left(t,{ }^{3} \mathrm{He}{ }^{58} \mathrm{Co}\right.$ reaction [30] was in good agreement with the $T=2 \mathrm{GT}$ strengths extracted from the ${ }^{58} \mathrm{Ni}\left({ }^{3} \mathrm{He}, t\right)$ measurement by using an analysis that distinguishes the $T=2$ and $T=1$ states [17]. In the analysis, the difference of the Clebsch-Gordan coefficients in the excitation of analog states with these different $T$ states in the proton inelastic scattering $\left[{ }^{58} \mathrm{Ni}\left(p, p^{\prime}\right)\right]$ and the $\left({ }^{3} \mathrm{He}, t\right)$ reaction. The consistency of the $T=2 \mathrm{GT}$ strength distribution with the ${ }^{58} \mathrm{Ni}\left(d,{ }^{2} \mathrm{He}\right)^{58} \mathrm{Co}$ reaction was also seen [31].

It is not often the case that absolute $B(\mathrm{GT})$ values are obtained for these transitions studied in the $(n, p)$-type CE reactions from the $\beta$-decay measurement of the analogous or corresponding transitions. It is noticed that by using the analogous relationship of the $T=1 \rightarrow 2$ GT transitions in the $\left({ }^{3} \mathrm{He}, t\right)$ and $\left(t,{ }^{3} \mathrm{He}\right)$ reactions, the absolute $B(\mathrm{GT})$ values can be determined for the transitions in the $\left(t,{ }^{3} \mathrm{He}\right)$ reaction.

\section{New $\beta$-decay experiments}

\subsection{Half-life measurement of ${ }^{54} \mathrm{Ni}$}

We now apply the "merged analysis" to the $A=54$ system, in which $T_{z}= \pm 1 \rightarrow 0$ mirror transitions are measured in the ${ }^{54} \mathrm{Fe}\left({ }^{3} \mathrm{He}, t\right){ }^{54} \mathrm{Co}$ reaction and the ${ }^{54} \mathrm{Ni} \beta$-decay. As we have seen, an accurate half-life plays an important role in the analysis. Therefore, for the $A=54$ system, we first had to seeking an accurate $T_{1 / 2}$ value. The $\beta$-decay of proton-rich nucleus ${ }^{54} \mathrm{Ni}$ was studied at the CRC, Louvain-la-Neuve. The ${ }^{54} \mathrm{Fe}\left({ }^{3} \mathrm{He}, 3 n\right){ }^{54} \mathrm{Ni}$ fusion evaporation reaction at the beam energy of $45 \mathrm{MeV}$ was used for the production of ${ }^{54} \mathrm{Ni}$. Nickel was selectively ionised in a laser ion source using a two-step ionisation scheme, and $A=54$ nuclei were selected by the Leuven isotope separator facility (LISOL) [32, 33, 34]. The mass separated ions were implanted in a tape system surrounded by three plastic $\beta$ detectors and two MINIBALL Ge detectors.

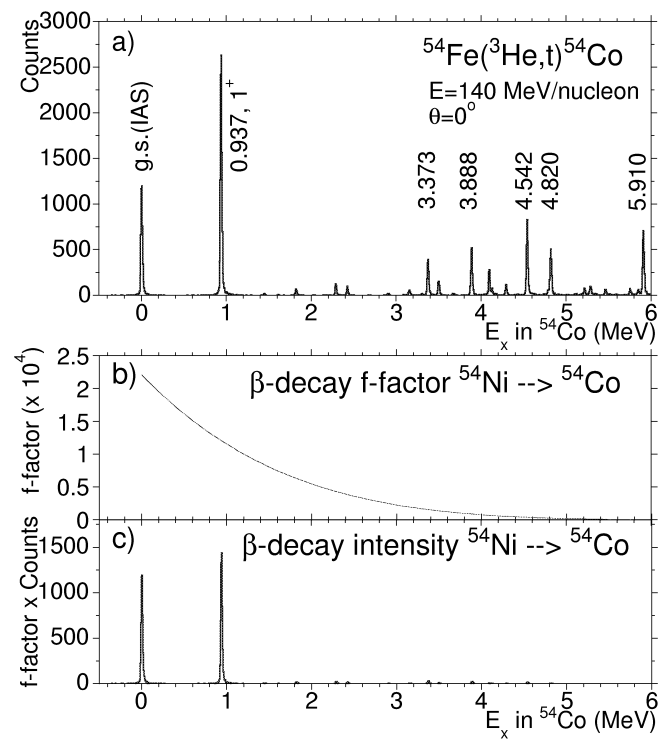

Figure 5: (a) $\mathrm{The}{ }^{54} \mathrm{Fe}\left({ }^{3} \mathrm{He}, t\right){ }^{54} \mathrm{Co}$ spectrum for events with scattering angles $\Theta \leq 0.5^{\circ}$. Major $L=0$ states, most probably the GT states, are indicated by their excitation energies in MeV. (b) The $f$-factor for the ${ }^{54} \mathrm{Ni} \beta$-decay, calculated from the decay $Q$-value of $8799(50) \mathrm{keV}$. (c) The estimated ${ }^{54} \mathrm{Ni} \beta$-decay energy spectrum that is obtained by multiplying the $f$-factor to the ${ }^{54} \mathrm{Fe}\left({ }^{3} \mathrm{He}, t\right)$ spectrum. Note that the IAS is stronger by a factor of $\approx 5$ in the real $\beta$-decay measurement due to the different coupling constants in the $\beta$-decay and the $\left({ }^{3} \mathrm{He}, t\right)$ reaction. 
The $T_{1 / 2}$ value of ${ }^{54} \mathrm{Ni}$ was determined by observing the exponential decay of the intensity of the $937 \mathrm{keV}$ delayed $\gamma$ rays from the first $J^{\pi}=1^{+}$GT state to the g.s. of ${ }^{54} \mathrm{Co}$. A preliminary analysis of the decay curve gave a half-life of $115 \pm 4 \mathrm{~ms}$ [35]. This value is longer than in Ref. [7] (106 $\pm 12 \mathrm{~ms}$ ), suggesting that the $B(\mathrm{GT})$ value should be smaller. It is important to note that the uncertainty has been reduced due to higher statistics.

The longer half-life and also the feeding to higher excited states found in the ${ }^{54} \mathrm{Fe}\left({ }^{3} \mathrm{He}, t\right){ }^{54} \mathrm{Co}$ reaction [26] made the GT transition strength to the $E_{x}=937 \mathrm{keV}$ state smaller. In the merged analysis, we obtain $B(\mathrm{GT})=0.46(8)$; this value is smaller by about $30 \%$ compared to the previous $\beta$-decay value of $0.68(16)$ [7]. The $T_{z}=+1 \rightarrow 0$ transition strength to this state has been measured in a ${ }^{54} \mathrm{Fe}(p, n)$ reaction at $E_{p}=135 \mathrm{MeV}$ [36]. Compared to their $B(\mathrm{GT})$ value of 0.74(5), which was derived from their own systematics, our new value is more than $35 \%$ smaller.

\subsection{Measurement of feeding ratio}

The $T_{z}=-1 \rightarrow 0 \beta$-decays of ${ }^{42} \mathrm{Ti},{ }^{46} \mathrm{Cr},{ }^{50} \mathrm{Fe}$ and ${ }^{54} \mathrm{Ni}$ and associated delayed $\gamma$ rays were studied. The experiment was performed as part of the RISING stopped beam campaign [37] at the FRagment Separator (FRS), GSI, Darmstadt. The experimental goals were to measure the halflives as well as the feeding ratios in these decays to derive $B(\mathrm{GT})$ values for comparison with the $T_{z}=+1 \rightarrow 0$ strengths measured in the corresponding $\left({ }^{3} \mathrm{He}, t\right)$ reactions. Beams of ${ }^{42} \mathrm{Ti},{ }^{46} \mathrm{Cr}$, ${ }^{50} \mathrm{Fe}$ and ${ }^{54} \mathrm{Ni}$ were produced by the fragmentation process from a primary $680 \mathrm{MeV} /$ nucleon ${ }^{58} \mathrm{Ni}$ beam of $0.1 \mathrm{nA}$ on a Be target. Each beam was well separated by the FRS facility and ions were implanted into an active stopper system consisting of three layers of double-sided silicon strip detectors (DSSDs). They were surrounded by the RISING $\gamma$-ray array composed of 15 EUROBALL cluster Ge detectors.

Due to the high production rate and the good detection efficiency of the RISING setup for ${ }^{54} \mathrm{Ni}$, as an example, high-energy delayed $\gamma$ rays could be seen [Fig. 6(b)] at the energies corresponding to the GT states observed in the ${ }^{54} \mathrm{Fe}\left({ }^{3} \mathrm{He}, t\right){ }^{54} \mathrm{Co}$ measurement [26]. A good symmetry is suggested for the $T_{z}= \pm 1 \rightarrow 0$ GT transitions, which supports the basis of the "merged analysis" of the $\beta$-decay and $\left({ }^{3} \mathrm{He}, t\right)$ results.
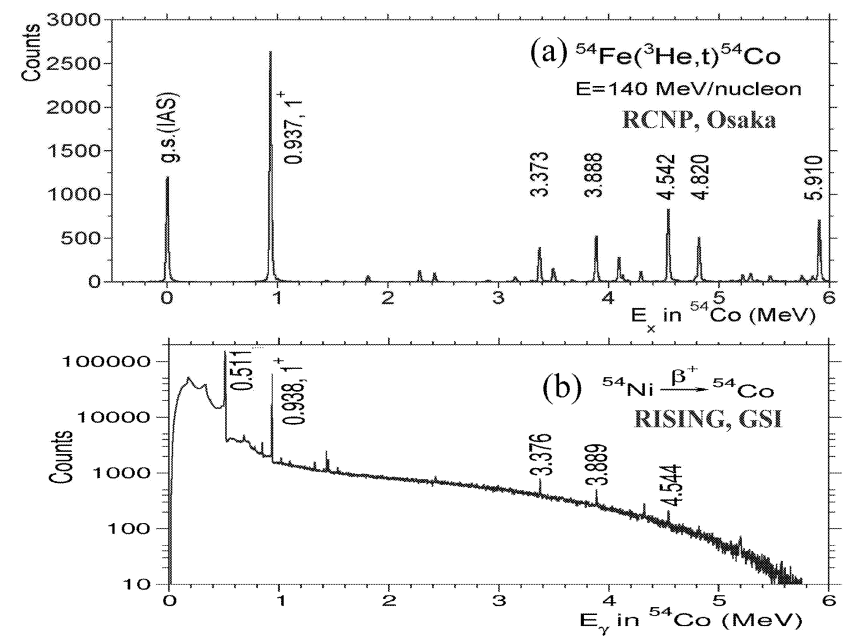

Figure 6: (a) $\mathrm{The}{ }^{54} \mathrm{Fe}\left({ }^{3} \mathrm{He}, t\right){ }^{54} \mathrm{Co}$ spectrum for events with scattering angles $\Theta \leq 0.5^{\circ}$. Major excited $L=$ 0 states are indicated by energies in $\mathrm{MeV}$. (b) On-line $\gamma$-ray spectrum at the RISING, GSI measured in coincidence with the $\beta$ particles from the ${ }^{54} \mathrm{Ni}$ decay. The existence of $\gamma$-ray peaks and CE-reaction peaks at corresponding energies suggests a good mirror symmetry of $T_{z}=-1 \rightarrow 0$ and $T_{z}=+1 \rightarrow 0$ GT transitions. 


\section{Summary and prospects}

With an improvement of the energy resolution in the $\beta^{-}$-type CE reaction $\left({ }^{3} \mathrm{He}, t\right)$, we started to see the details of the GT transitions; fine structures of GT excitations, even those of GT giant resonances, can now be studied. For $s d$-shell nuclei, $B(\mathrm{GT})$ values of multiple GT transitions are studied for $T_{z}=-1 / 2 \rightarrow+1 / 2$ and $T_{z}=-1 \rightarrow 0$ transitions in $\beta^{+}$decays. The analogous $T_{z}=+1 / 2 \rightarrow-1 / 2$ and $T_{z}=+1 \rightarrow 0$ transitions were studied in ( $\left.{ }^{3} \mathrm{He}, t\right)$ reactions. By comparing these analogous transitions, a good proportionality between the cross-sections at $0^{\circ}$ and $B(\mathrm{GT})$ values were observed in the $\left({ }^{3} \mathrm{He}, t\right)$ reaction at $140 \mathrm{MeV} /$ nucleon.

Owing to the proportionality, the $B(\mathrm{GT})$ values can be deduced up to high excitation region once a standard $B(\mathrm{GT})$ value is provided by the $\beta$-decay. However, in the $p f$-shell region, the $\beta$-decay $B(\mathrm{GT})$ values have lager uncertainties; only $T_{1 / 2}$ values were relatively reliable. We introduced the "merged analysis" to overcome this difficulty and to determine the absolute $B(\mathrm{GT})$ values. We performed ( $\left.{ }^{3} \mathrm{He}, t\right)$ measurements on $T_{z}=+1 p f$-shell nuclei to study $T_{z}=+1 \rightarrow 0$ GT transitions. With an energy-resolution of about $30 \mathrm{keV}$, discrete GT states were identified. The unknown energy spectra of the $T_{z}=-1 \rightarrow 0 \beta$-decay of exotic nuclei were estimated by multiplying the $f$-factor calculated from the $Q$-value of the decay. By further combining the half-life values obtained in the $\beta$-decay measurements, absolute values of GT transition strengths $B(\mathrm{GT})$ were derived.

This merged analysis for the determination of absolute GT strengths can be extended to other $T=1$ systems and also to $T=2$ and even higher $T$ systems, thus allowing to deduce the GT strength distributions in proton-rich exotic nuclei. The uncertainty of the obtained GT strength is mainly due to the ambiguities in the $T_{1 / 2}$ and the decay $Q$ value. Therefore, efforts to obtain accurate $T_{1 / 2}$ values and also the feeding ratios of GT transitions for proton-rich nuclei have been performed. New attempts at ISOL facilities (e.g. [38]) as well as at fragment-separator facilities (e.g. [39], [40]) are also in progress. In addition, precise $Q$-value (mass) measurements that can be performed at trap facilities [41] would bring more accurate $f$-factors. A better knowledge on them will make the merged analysis more fruitful as the means to determine the GT strengths in proton-rich exotic $p f$-shell nuclei, which are needed to deduce the astrophysical transition rates under extreme conditions.

The high-resolution $\left({ }^{3} \mathrm{He}, t\right)$ experiments were performed at RCNP, Osaka University. The author is grateful to the RCNP accelerator group, especially to Professors Hatanaka and Saito. He is grateful to Dr. B. Rubio (Valencia) for various discussions. This work was supported in part by Monbukagakusho, Japan under Grant No. 15540274 and No. 18540270, and also by the Japan-Spain collaboration program by JSPS and CSIC.

\section{References}

[1] A. Heger et al., Phys. Rev. Lett. 86, 1678 (2001).

[2] A.B. Balantekin and G.M. Fuller, J. Phys. G: Nucl. Part. Phys. 29, 2513 (2003).

[3] K. Langanke et al., Phys. Rev. Lett. 93, 202501 (2004).

[4] J.C. Hardy and I.S. Towner, Phys. Rev. C 71, 055501 (2005), ibid. Nucl. Phys. News 16, 11 (2006).

[5] T.K. Onishi et al. Phys. Rev. C 72, 024308 (2005).

[6] V.T. Koslowsky et al. Nucl. Phys. A624, 293 (1997). 
[7] I. Reusen et al. Phys. Rev. C 59, 2416 (1999).

[8] A. Jokinen et al. Eur. Phys. J. A 3, 271 (1998).

[9] T.N. Taddeucci et al., Nucl. Phys. A469, 125 (1987).

[10] WEB page htip://www.rcnp.osaka-u.ac.jp

[11] T. Wakasa et al., Nucl. Instrum. Methods Phys. Res. A 482, 79 (2002).

[12] M. Fujiwara et al., Nucl. Instrum. Methods Phys. Res. A 422, 484 (1999).

[13] Y. Fujita et al., Nucl. Instrum. Methods Phys. Res. B 126, 274 (1997).

[14] Y. Fujita et al., Nucl. Phys. A687, 311c (2001).

[15] H. Fujita et al., Nucl. Instrum. Methods Phys. Res. A 484, 17 (2002).

[16] J. Rapaport and E. Sugarbaker, Annu. Rev. Nucl. Part. Sci. 44, 109 (1994).

[17] H. Fujita et al., Phys. Rev. C 75, 034310 (2007).

[18] A. Bohr and B. Mottelson, Nuclear Structure (Benjamin, New York, 1975), Vol. 2, Chap. 6.

[19] Y. Fujita et al., Phys. Rev. C 67, 064312 (2003).

[20] R. Zegers et al., Phys. Rev. C 74, 024309 (2006).

[21] Y. Fujita et al., Phys. Rev. C 59, 90 (1999).

[22] Y. Fujita et al., Phys. Rev. C 66, 044313 (2002).

[23] R. Zegers et al., Phys. Rev. Lett. 99, 202501 (2007).

[24] Y. Fujita et al., Phys. Rev. C 75, 057305 (2007).

[25] J.W. Watson and Q.Q. Du, Nucl. Phys. A687, 32c (2001).

[26] T. Adachi et al., Nucl. Phys. A788, 70c (2007).

[27] T. Adachi et al., Phys. Rev. C 73, 024311 (2006).

[28] Y. Fujita et al., Phys. Rev. Lett. 95, 212501 (2005).

[29] G.W. Hitt et al., Contribution to this NIC-X Symposium.

[30] A.L. Cole et al., Phys. Rev. C 74, 034333 (2006).

[31] M. Hagemann et al., Phys. Rev. C 71, 014606 (2005).

[32] Yu. Kudryavtsev et al., Nucl. Instrum. Meth. Phys. Res. B 179, 412 (2001).

[33] M. Facina et al., Nucl. Instrum. Meth. Phys. Res. B 226, 401 (2004), and references therein.

[34] Yu. Kudryavtsev et al., Nucl. Instrum. Meth. Phys. Res. B 114, 350 (1996).

[35] B. Rubio, F. Molina, Y. Fujita, W. Gelletly et al., to be submitted.

[36] B.D. Anderson et al., Phys. Rev. C 41, 1474 (1990).

[37] "Stopped Beam" RISING experimental campaign at GSI, spokespersons: P.H. Regan, J. Gerl, and H.J. Wollersheim.

[38] B. Rubio et al., experimental proposal at the CRC, Louvain la Neuve (2007).

[39] C. Dossat, B. Blank et al., Nucl. Phys. A 792, 18 (2007).

[40] Y. Fujita, B. Rubio, W. Gelletry et al., experimental proposal "E556" at GANIL, Caen, France.

[41] See, for example, the contribution by K. Blaum to this NIC-X symposium. 